\title{
USO COMBINADO DO MÉTODO DE MONTE CARLO E CRITÉRIOS DE DECISÃO EM CONDIÇÕES DE INCERTEZA
}

\section{COMBINED USE OF MONTE CARLO METHOD AND DECISION CRITERIA IN CONDITIONS OF UNCERTAINTY}

\author{
Lucas Humaita Blitzkow da Silva ${ }^{1}$, Andréa Ryba ${ }^{1, \bigotimes}$, Marcelo Kaminski Lenzi ${ }^{1}$ \\ ${ }^{1}$ Universidade Federal do Paraná, Curitiba, Paraná, Brasil \\ andrea.ryba@ufpr.br
}

Recebido: 12 dezembro 2018 / Aceito: 29 março 2019/ Publicado: 11 julho 2019

\begin{abstract}
In a world where investing accompanies people's lives, the choice of the best application is of great importance. However, the uncertainties closely linked to the predictions of the future make some decisions not so simple to be made. This work seeks to support the decisionmaking process regarding investment projects considering the inherent uncertainties. Using the Net Present Value (NPV) concept, coupled with the Monte Carlo Method to simulate possible future scenarios, the uncertainties can then be modeled. With the help of Decision Criteria in Classical Uncertainty Conditions (Maximin, Maximax, Hurwicz, Savage and Laplace) and novel approaches (Modified Hurwicz and Amplitude Model), such scenarios can be evaluated, even without statistical probabilities of occurrence being attributed to them, and an indication of the best investment option is presented. Thus, the investment analysis, with uncertainties modeled and a direct response being presented to the investor, even considering a future without attributions of probabilities for their possible scenarios, was satisfactory. As a differential, the possibility of comparing investments with the same duration and / or different durations should be highlighted.
\end{abstract}

Keywords: Investment. Uncertainties. Monte Carlo method. Decision Criteria in Conditions of Uncertainty.
RESUMO. Em um mundo onde investir é uma realidade que acompanha a vida das pessoas, a escolha em que se investir tem uma grande importância. No entanto, as incertezas intimamente ligadas às previsões do futuro fazem com que algumas decisões não sejam simples. Este estudo busca amparar o processo de tomada de decisão sobre projetos de investimentos considerando as incertezas inerentes ao processo. Utilizando-se do conceito de VPL, unido ao Método de Monte Carlo para simulação de possíveis cenários futuros, as incertezas podem, então, serem modeladas. E com o auxílio de Critérios de Decisão em Condições de Incerteza clássicos (Maximin, Maximax, Hurwicz, Savage e Laplace) e inovadores (Hurwicz Modificado e Modelo de Amplitude), tais cenários podem ser avaliados, mesmo sem que sejam atribuídas probabilidades estatísticas de ocorrência a eles, sendo apresentada uma indicação da melhor opção de investimento. Desta forma, a análise de investimentos, com incertezas modeladas e uma resposta direta sendo apresentada ao investidor, mesmo considerando um futuro sem atribuições de probabilidades para seus possíveis cenários, foi satisfatória. Há que se ressaltar ainda, como diferencial, a possibilidade de comparar investimentos com mesmos períodos de duração e/ou durações diferentes.

Palavras-chave: Investimento. Incertezas. Método de Monte Carlo. Critérios de Decisão em Condições de Incerteza 


\section{INTRODUÇÃO}

Em mercados onde investimentos muitas vezes são caracterizados por grandes quantias de dinheiro, as decisões são os divisores de águas entre a lucratividade e o prejuízo, entre melhorar a vida das pessoas ou manter a realidade como está, quando não a piorar ainda mais. Projetos com grandes volumes de capital e que tem impacto na vida de muitas pessoas precisam prioritariamente ser assertivos. Assim, com a devida reflexão, a necessidade de planejar investimentos apresenta-se como alternativa para evitar desperdícios e/ou mal alocação de recursos, tanto no ponto de vista individual como no coletivo (empresas e governos).

Um ponto importante a ser considerado neste contexto é o tratamento do tempo futuro. A necessidade de gerar cenários que simulem acontecimentos (previsões de desembolsos e recebimentos) para a quantificação das informações e decorrente comparação entre alternativas é fundamentada em premissas. Isto significa que aspectos que são incertos, por mais que possam ser previsíveis, precisam ser colocados como certos (O’Donnell et al., 2002). É neste ponto que se apresenta a problemática da situação: a possibilidade do futuro não ser condizente com o que foi proposto. Os resultados podem variar desde pequenas possíveis adaptações necessárias até a inviabilização do empreendimento com prejuízos nas mais diversas escalas.

Para que seja possível lidar com tal problema, Galesne et al. (1999) apresentam dois cenários: o de futuro indeterminado e o de futuro determinado probabilisticamente. Tal divisão serve para, considerando que o porvir é incerto, separar as características de tal incerteza. Enquanto, no primeiro caso, não é possível associar uma distribuição de probabilidades aos acontecimentos estudados, no segundo tal associação torna-se possível. A distinção apresentada tem como objetivo contrapor o clássico critério de Knight, o qual afirma que risco e incerteza são conceitos diferentes. Enquanto ao primeiro pode-se atribuir uma distribuição de probabilidades, ao segundo não. (Gaslene et al., 1999)

Considerando um futuro determinável, com o auxílio de ferramentas estatísticas, torna-se mais seguro fazer previsões e usá-las em análises de investimentos. Já, considerando um futuro indefinido, a tratativa das incertezas nas previsões é uma etapa fundamental para a tomada de decisão, num cenário que se desconhece a distribuição de probabilidades aleatória (Chobot, 2013). 
Nesse sentido, a quantidade de incertezas, muitas vezes, aumenta proporcionalmente a complexidade dos projetos fazendo com que tais decisões demandem ainda mais acurácia em um ambiente da mesma forma mais hostil para tal. Assim, uma metodologia de análise de investimentos, capaz de inserir tais incertezas em sua modelagem e que possua, ainda, aplicabilidade nos mais diversos campos (engenharias, administração ou economia, por exemplo) pode ser de grande auxílio para diversos e diferentes tipos de profissionais. Ainda mais, considerando os questionamentos que alguns deles acabam por criar em relação a suas próprias projeções, pois com o grande número de incertezas, não possuem confiança de que essas são ou não condizentes com a realidade.

Diante disso, essa pesquisa tem por objetivo apresentar uma metodologia que permita a escolha entre diferentes opções de investimentos através de critérios de decisão em situações de incerteza (futuro indeterminado) com a utilização do Método de Monte Carlo como ferramenta de criação de possíveis cenários, permitindo, assim, ao investidor a possibilidade de tomada de decisão mesmo sem conhecer as probabilidades dos eventos futuros.

Tal metodologia surge como uma opção entre metodologias que usam apenas o Método de Monte Carlo para avaliação de risco, como apresentado por Han et al. (2017) e também por Buchner (2017), ou os Critérios de Decisão em Condições de Incerteza (Garcia et al., 2010; Pažek and Rozman, 2009; Groenewald and Pretorius, 2011; Gaspars-Wieloch, 2014) para escolha em cenários futuros já simulados. A união proposta aqui atinge, de forma diferenciada, a possibilidade de simulação dos cenários futuros probabilisticamente indefinidos com o Método de Monte Carlo, com a possibilidade da escolha da melhor estratégia de investimento e com Critérios de Incerteza ideais para utilização em tais condições.

\section{REVISÃO BIBLIOGRÁFICA}

\subsection{PROJETOS DE INVESTIMENTOS}

Num contexto empresarial, Laurencel \& Rezende Filho (2013) acreditam que investir é comprometer capital, buscando criação de valor. Não só isso, mas também, fazê-lo por um determinado tempo e com a esperança de se obter uma rentabilidade. Já Ryba et al. (2016), 


\section{REVISTA LATINO-AMERICANA DE INOVAÇÃO E ENGENHARIA DE PRODUÇÃO}

analisando no âmbito industrial, definiu investimento como um gasto focado na melhoria do processo de produção e, consequentemente, um projeto de investimento relaciona-se a um amplo estudo abrangendo, como também foi abordado por Casarotto Filho \& Kopittke (2006), três critérios: econômicos, financeiros e os imponderáveis.

Dentre os critérios citados, o de maior interesse para essa pesquisa são os critérios econômicos, já que são os responsáveis por avaliar a rentabilidade de um investimento. São exemplos desses métodos: o método valor anual, do valor presente, da taxa interna de retorno e do tempo de retorno ou recuperação (Payback), dentre outros. (Ryba et al., 2016)

Dos métodos citados anteriormente, aquele do Valor Presente Líquido com o auxílio da taxa mínima de atratividade serão utilizados como parte da metodologia objetivo de estudo desta pesquisa. O método do Valor Presente Líquido foi escolhido em virtude de ser o mais amplamente utilizado, conforme reportado por Mellichamp (2013). Conforme reportado por Sharma (2015), esse método consiste em trazer para o presente todas as entradas (ou recebimentos) do fluxo de caixa característico do projeto em análise e todas as saídas de caixa (ou gastos) até o tempo presente, considerando a TMA como taxa de juros referência para tal, conforme pode ser observado na Equação 01:

$$
V P L=\left[\sum \frac{F C_{n}}{(1+T M A)^{n}}\right]-C
$$

onde $\mathrm{n}$ é o período, $\mathrm{FC}_{\mathrm{n}}$ é o fluxo de caixa das entradas (valores positivos) e das saídas (valores negativos) no período n e C é o investimento inicial.

A Taxa Mínima de Atratividade (TMA) permite comparar um fluxo de caixa em análise com um outro investimento referência de atratividade mínima admissível, que já seja de prática usual da empresa, conforme mencionado por Camargo (2007). A correta determinação dessa taxa deve-se basear em três pilares: a liquidez, relacionada ao tempo em que o recurso fica indisponível ao investidor para que possa se valorizar; o risco ou nível de incerteza ou probabilidade de o investimento não ser atrativo da maneira esperada; e a sua rentabilidade, que representa quanto o projeto pode render ao investidor. No entanto, a principal questão é que esses três aspectos geralmente funcionam em direção oposta; por exemplo, quanto maior a lucratividade (característica desejada), maior o risco (característica indesejada); quanto maior a rentabilidade (característica desejada), menor a liquidez (característica indesejada). 


\subsection{MÉTODO DE MONTE CARLO}

O Método de Monte Carlo, criado por J. von Neumann e S. Ulam e apresentado em 1949 no artigo “The Monte Carlo method”, é um método de solução numérica de problemas essencialmente baseado na simulação de variáveis aleatórias (Sobol, 1972). Embora envolva amostragem aleatória, o seu principal objetivo é estimar um valor determinístico (Brandimarte, 2014).

Variável aleatória, por sua vez, é “a variável que, não assumindo necessariamente um valor preciso, pode assumir diversos valores, cada um com uma certa probabilidade" (Sobol, 1972). Podem ser, ainda, classificadas como: discretas, contínuas e/ou normais. As variáveis aleatórias discretas são aquelas que podem assumir qualquer valor em um conjunto finito. As contínuas se destacam por existir um intervalo que compreende seu domínio, ou seja, pode apresentar valores dentro deste e ter associada a si uma função de densidade de probabilidade. Isto significa que os valores possíveis de serem assumidos pela variável tem uma probabilidade de ocorrer e esta pode ser representada por uma função (Sobol, 1972). Por fim, ainda é possível classificar uma variável aleatória como sendo normal. Esta tem como característica obedecer, dentro de um domínio que varia do menos infinito para o mais infinito, a uma função de densidade representada pela Equação (02):

$$
p(x)=\frac{1}{\sqrt{2 \pi \sigma}} e^{\frac{-(x-a)^{2}}{2 \sigma^{2}}}
$$

onde a e $\sigma$ são os parâmetros numéricos que a individualizam. (Sobol, 1972).

O Teorema Central do Limite, formulado por Laplace, afirma que a somatória de diversas variáveis aleatórias independentes gerará, aproximadamente, uma distribuição normal (Sobol, 1972).

O Método de Monte Carlo é muito difundido e utilizado em diversas pesquisas na área de análise de investimento. Em Lima et al. (2008), o método de Monte Carlo, assim como neste trabalho, é apresentado de forma a fazer parte de uma metodologia de análise de investimentos e esta, por sua vez, é aplicada a um estudo de caso, também hipotético, relacionado a fábricas de refrigerantes.

Outra aplicação do método, reportado por Garcia et al. (2010), se dá em um estudo de caso envolvendo a Companhia Vale do Rio Doce, verificando e confirmando a 
aplicabilidade deste na previsão dos custos de produção e sua utilidade no auxílio do processo decisório. Já Souza et al. (2016), por sua vez, aplicaram o Método de Monte Carlo para avaliação de risco no sistema de produção de soja no estado do Pará e Junior et al (2017), utilizam o método com objetivo de comparar custos de produção, expectativas de retorno e riscos na exploração comercial de 1 (um) hectare de erva-mate com cultivo tradicional ou orgânico.

Han et al. (2017) aplicou a técnica de simulação de Monte Carlo para avaliar os riscos globais de projetos de investimento de parcerias público-privadas na implantação de pedágios em rodovias. Já Buchner (2017) utilizou o referido método para simular a dinâmica de fluxos de caixa dos fundos de ativos privados, do inglês private equity.

Os exemplos anteriores são apenas alguns dos diversos trabalhos e pesquisas que já foram e são realizadas utilizando o Método de Monte Carlo, demonstrando não só sua importância como também sua utilidade nas mais diversas áreas de conhecimento.

\subsection{CRITÉRIOS DE DECISÃO EM CONDIÇÕES DE INCERTEZA}

As incertezas estão presentes em todo tipo de projeto de investimento e os que envolvem a aplicação de capital, aqui estudados, não são exceções. Segundo Casarotto Filho \& Kopittke (2006) existem três alternativas para solução de problemas que envolvem incertezas. São elas: o uso de regras, ou critérios de decisão vinculados à matrizes de decisão; análise de sensibilidade e simulação.

A metodologia de análise de investimento proposta neste estudo está intimamente ligada a duas dessas alternativas. Primeiramente relacionada ao uso de simulações, cuja ferramenta, o Método de Monte Carlo, já foi apresentada, e ainda o uso de Matrizes de Decisão aliadas aos Critérios de Decisão em Condições de Incerteza.

Além dos estudos apresentados por Ramírez \& García (2001), Ramírez \& García (2003) e Pérez et al. (2015) relacionados aos Critérios de Decisão em Condições de Incerteza, Fonseca (2003) realizou um estudo em fôrma de revisão bibliográfica a fim de descrever as principais etapas de avaliação de investimentos no atual contexto de globalização e inseriu os Critérios em Incerteza como uma ferramenta de auxílio à tomada de decisão.

Martins (2012), por sua vez, realizou uma pesquisa com o intuito de defender a ideia 
de que, com o uso correto de modelos matemáticos, riscos e incertezas poderiam ser devidamente consideradas e medidos de forma consciente.

Os trabalhos mencionados demonstram que existem pesquisadores que acreditam na aplicação dos Critérios de Incerteza na modelagem matemática de análises de investimento. Sendo que, nas condições de futuro indeterminado probabilisticamente, Casarotto Filho \& Kopottke (2006), cujo trabalho é uma referência na área da análise de investimentos, indicam tais critérios.

A seguir serão apresentados os conceitos relativos à Matriz de Decisão e à Critérios clássicos de Decisão em Condições de Incerteza (Maximin, Maximax, Hurwicz, Savage e Laplace) seguindo o apresentado por Casarotto Filho \& Kopittke (2006). Depois, serão apresentados dois critérios mais recentemente propostos para a utilização neste tipo de situação (Hurwicz Modificado e o Modelo de Amplitude).

\subsubsection{Matriz de Decisão}

Segundo Casarotto Filho \& Kopittke (2006), as Matrizes de Decisão, no âmbito econômico, são tabelas, cujo valor apresentado se refere a consequência da escolha de uma estratégia de investimento com ocorrências de determinados cenários futuros.

$\mathrm{Na}$ metodologia apresentada neste trabalho os valores representados no interior das células que compõem a Matriz de Decisão serão valores calculados de VPL para diferentes cenários e diferentes estratégias de investimento.

Com a Matriz de Decisão devidamente organizada e apresentada é possível agora, ao investidor, a aplicação dos Critérios de Decisão em Condições de Incerteza para a escolha de qual projeto será executado.

\subsubsection{Maximin}

O Critério Maximin consiste em escolher a opção de investimento que apresente o maior valor entre os menores valores. Para tal, para cada alternativa deve ser extraído o cenário mais pessimista, ou seja, aquele que apresenta o menor VPL. Na sequência o pior de cada alternativa é comparado com os piores das demais e o que for mais alto indicará qual o plano que será definido para investimento. Esse é chamado de critério do pessimista, pois 
nele é considerado o pior cenário de cada opção de investimento na consideração da escolha entre elas (Casarotto Filho \& Kopittke, 2006).

\subsubsection{Maximax}

Conforme reportado por Casarotto Filho \& Kopittke (2006) e por Pažek \& Rozman (2009), o Critério do Maximax, considera os cenários mais otimistas, ou seja, mais favoráveis para cada uma das opções de investimento. Portanto, compara entre si os maiores VPL's obtidos na modelagem de cada estratégia e indica a escolha do maior deles. De forma oposta ao Maximin, esse critério utiliza-se dos cenários mais otimistas para direcionar a opção a ser adotada.

\subsubsection{Hurwicz}

Segundo Pažek \& Rozman (2009), o Critério de Hurwicz faz uma ponderação entre os extremos pessimistas e otimistas. Por sua vez, calcula um VPL representativo para cada uma das opções de investimento. Para isso, utiliza-se de um coeficiente relacionado ao nível de otimismo do investidor e dos valores máximos e mínimos de VPL de cada alternativa.

O coeficiente mencionado nada mais é do que a maneira de inserir pesos em uma média ponderada entre o valor do cenário mais otimista e do mais pessimista de cada estratégia de investimento. Portanto, quanto mais otimista for o investido em relação a opção analisada, maior peso terá o maior VPL modelado para esta alternativa em detrimento do cenário mais pessimista.

A seguir, com a Equação (03), também reportada por Pažek \& Rozman (2009) e já adaptada a nomenclatura dessa pesquisa, calcula-se o VPL representativo deste critério:

$$
\mathrm{VPL}_{\mathrm{REP}}=\alpha \cdot \mathrm{VPL}_{\mathrm{MÁX}}+(1-\alpha) \cdot \mathrm{VPL}_{\mathrm{MÍN}}
$$

onde o VPLREP é o VPL representativo, $\alpha$ é o coeficiente responsável por representar o otimismo do investidor quanto ao projeto, com valor variando entre 0 e 1 sendo que quanto

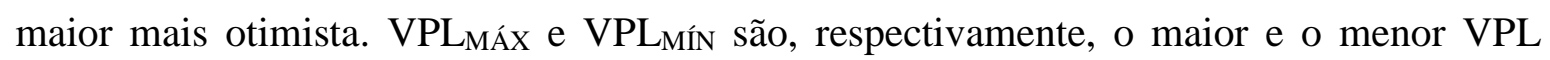
simulado para determinada alternativa de investimento. 


\subsubsection{Savage}

Para a utilização do critério de Savage, também conhecido como modelo de arrependimento (García et al. 2014), é calculada uma Matriz de Arrependimento. Esta representa a diferença entre o maior VPL entre todos os cenários de uma dada opção de investimento e cada um dos demais, sendo que tal procedimento é repetido para todas as opções de investimento.

A opção escolhida, segundo este critério, será a que apresentar um menor valor de arrependimento máximo. Em outras palavras, da Matriz de Arrependimento são extraídos o maior valor de cada investimento. O projeto que possuir o menor entre esses deverá ser escolhido.

\subsubsection{Laplace}

O Critério de Laplace, diferentemente dos anteriores, considera no cálculo do VPL representativo de cada estratégia de investimento, não apenas um ou dois valores de VPL de cenários específicos, mas também todos os outros.

Isso ocorre pois, nesta metodologia calcula-se a média dos VPL's de todos os cenários de cada projeto e estas, por sua vez, são comparadas, sendo que a escolhida será a de maior valor absoluto.

A seguir a Equação (04) relativa ao critério, também apresentada por Pérez et al., 2015 e já adaptada a nomenclatura desse trabalho:

$$
\mathrm{VPL}_{\mathrm{REP}}=\frac{\Sigma \mathrm{VPL}_{i}}{n}
$$

onde n é o número de cenários simulados e $\mathrm{VPL}_{\mathrm{i}}$ representa cada um dos valores de VPL para os diferentes cenários simulados de uma opção de investimento.

\subsubsection{Hurwicz Modificado}

O Critério de Hurwicz Modificado, assim como o Critério de Hurwicz utiliza um coeficiente responsável por representar o otimismo do investidor quanto ao investimento em forma de pesos para uma média ponderada. Porém, neste caso, não são utilizados os valores 


\section{REVISTA LATINO-AMERICANA DE INOVAÇÃO E ENGENHARIA DE PRODUÇÃO}

máximo e mínimo para o cálculo do VPL representativo do projeto (Pérez et al., 2015).

A modificação do critério é o cálculo de duas médias para que estas entrem na média ponderada característica do Modelo de Hurwicz. Com os valores de VPL de cada opção de investimento, rearranjados de forma crescente, a primeira metade (os 50\% maiores valores de VPL) é utilizada na média que representa o valor máximo do modelo tradicional, enquanto, de maneira análoga, o mesmo ocorre com a segunda metade (os 50\% menores valores de VPL) e o valor mínimo do modelo tradicional (Pérez et al., 2015).

\subsubsection{Modelo de Amplitude}

O Modelo de Amplitude, por sua vez, é um Critério que procura considerar no cálculo do VPL representativo de cada uma das alternativas de investimento a amplitude dos valores apresentados nos diversos cenários modelados (Ramírez \& García, 2001; Pérez et al., 2015).

Tal consideração funciona como uma penalização, sendo que quanto maior a amplitude dos valores, maior será o desconto no VPL representativo calculado e consequentemente menos atrativo o investimento se apresenta (Ramírez \& García, 2001; Pérez et al., 2015).

A equação de cálculo do VPL representativo para cada opção de investimento, no caso considerado por Ramírez \& García (2001) e Pérez et al. (2015) de maximização, é apresentada pela Equação (05), como segue:

$$
\mathrm{VPL}_{\mathrm{REP}}=\mathrm{VPL}_{\text {médio }}+\left[\beta \cdot \mathrm{VPL}_{\text {médio }}-(1-\beta) \cdot A\right]
$$

onde VPLmédio é a média dos VPL's considerando todos os cenários onde a alternativa é simulada e A é a amplitude, calculada como a diferença entre o maior VPL observado e o menor. O coeficiente $\beta$, por sua vez, foi estudado por Ramírez \& García (2003), e foi definido como ideal a utilização deste na faixa de 0,80 .

Os diferentes Critérios de Decisão em Condição de Incerteza têm como característica em comum indicarem uma opção de investimento como aquela que deve ser escolhida pelo investidor. Essa característica será essencial para que o objetivo da metodologia de análise de investimento, aqui em estudo, de fornecer uma resposta direta, mesmo com a consideração de um futuro indeterminado probabilisticamente seja atingida. 


\section{METODOLOGIA}

Tal metodologia foi aplicada de forma a minimizar as dificuldades e tomar as devidas decisões, de forma a escolher, por exemplo, entre 3 opções de investimento. As opções de investimento, apresentadas na Tabela 1, tratam de casos hipotéticos que são compatíveis com o que pode ser encontrado na realidade de diferentes mercados industriais.

TABELA 1 - PARÂMETROS DOS INVESTIMENTOS

\begin{tabular}{lccc}
\hline \multicolumn{1}{c}{ Parâmetros } & Investimento 1 & Investimento 2 & Investimento 3 \\
\hline Número de Períodos & 21 meses & 18 meses & 24 meses \\
\hline Investimento Inicial (Média) & $\mathrm{R} \$ 2.000 .000,00$ & $\mathrm{R} \$ 2.000 .000,00$ & $\mathrm{R} \$ 2.000 .000,00$ \\
\hline $\begin{array}{l}\text { Investimento Inicial } \\
\text { (Desvio Padrão) }\end{array}$ & $\mathrm{R} \$ 100.000,00$ & $\mathrm{R} \$ 75.000,00$ & $\mathrm{R} \$ 125.000,00$ \\
\hline Custo Despesas Fixas & $\mathrm{R} \$ 80.000,00$ & $\mathrm{R} \$ 130.000,00$ & $\mathrm{R} \$ 20.000,00$ \\
\hline Demanda por Período (Média) & 18.000 unidades & 10.000 unidades & 33.000 unidades \\
\hline $\begin{array}{l}\text { Demanda por Período (Desvio } \\
\text { Padrão) }\end{array}$ & 1.575 unidades & 750 unidades & 3.300 unidades \\
\hline $\begin{array}{l}\text { Preço de Venda Unitário } \\
\text { (Média) }\end{array}$ & $\mathrm{R} \$ 40,00$ & $\mathrm{R} \$ 100,00$ & $\mathrm{R} \$ 15,00$ \\
\hline $\begin{array}{l}\text { Preço de Venda Unitário } \\
\text { (Desvio Padrão) }\end{array}$ & $\mathrm{R} \$ 3,50$ & $\mathrm{R} \$ 7,50$ & $\mathrm{R} \$ 1,50$ \\
\hline $\begin{array}{l}\text { Custo Matéria Prima Unitário } \\
\text { (Média) }\end{array}$ & $\mathrm{R} \$ 15,00$ & $\mathrm{R} \$ 30,00$ & $\mathrm{R} \$ 3,00$ \\
\hline $\begin{array}{l}\text { Custo Matéria Prima Unitário } \\
\text { (Desvio Padrão) }\end{array}$ & $\mathrm{R} \$ 0,94$ & $\mathrm{R} \$ 1,50$ & $\mathrm{R} \$ 0,23$ \\
\hline $\begin{array}{l}\text { Custo Fornecedor Unitário } \\
\text { (Média) }\end{array}$ & $\mathrm{R} \$ 8,00$ & $\mathrm{R} \$ 30,00$ & $\mathrm{R} \$ 5,00$ \\
\hline $\begin{array}{l}\text { Custo Fornecedor Unitário } \\
\text { (Desvio Padrão) }\end{array}$ & $\mathrm{R} \$ 0,50$ & $\mathrm{R} \$ 1,50$ & $\mathrm{R} \$ 0,38$ \\
\hline
\end{tabular}

FONTE: Os autores (2018)

Os referidos valores, apresentados na Tabela 1, são ligados a dois valores cada: valor médio e um valor de desvio padrão. Nesta metodologia de análise, esses valores são usados no Método de Monte Carlo para, através de distribuições normais de probabilidade, gerar dez mil números aleatórios para cada parâmetro de cada alternativa, com exceção do número de períodos e do custo fixo que se mantiveram fixos nos valores apresentados. 


\section{REVISTA LATINO-AMERICANA DE INOVAÇÃO E ENGENHARIA DE PRODUÇÃO}

Os parâmetros utilizados no Método de Monte Carlo se repetiram em cada um dos períodos relativos ao seu investimento e para cada um destes foram gerados dez mil valores. Para exemplificar: O Preço de Venda Unitário da Opção de Investimento 1, cujo valor médio é de $\mathrm{R} \$ 40,00$ e o desvio padrão R \$3,50, gerou, pelo Método de Monte Carlo, dez mil valores para cada um dos 21 períodos de duração deste investimento. Portanto, para a criação de dez mil possíveis alternativas de preços de vendas, em cada um dos 21 períodos de duração do investimento, foram gerados 210.000 valores de preço de venda aleatoriamente, respeitando a distribuição normal caracterizada pelos valores de média e desvio padrão empregados.

$\mathrm{Na}$ sequência, considerou-se que, quanto menor o conhecimento do pesquisador quanto ao mercado que deseja investigar, maior será o nível de incerteza atribuído aos parâmetros e, consequentemente, maior será o desvio padrão relacionado a eles, ou seja, foi atribuída uma correlação entre o valor do desvio padrão de cada parâmetro e níveis de incerteza.

Aqui se faz a ressalva de que, por se tratar de um problema em condições de incerteza (futuro não determinável probabilisticamente) as atribuições de média e desvio padrão, utilizadas para gerar a simulação, são intimamente ligadas a um conhecimento não baseado unicamente, em dados estatísticos (caso existam), do investidor sobre o mercado. Caso contrário o problema seria tratado como definiu Galesne et al. (1999) como em um futuro determinável probabilisticamente, o que não é o objetivo desta metodologia. Tal procedimento foi possível com a criação de uma formulação que correlacionasse o nível de incerteza do investidor com o desvio padrão do parâmetro analisado.

O referido nível de incerteza foi dividido em 2 parâmetros: a incerteza global relativa ao tipo de investimento (IG), que é função dos tipos de mercado e produto que estão em análise; e a incerteza específica (IE), característica do parâmetro que está sendo analisado (preço de venda, demanda etc.). Ambos os valores representados em termos percentuais em relação à média.

Além disso, foram atribuídos pesos para cada um dos níveis de incerteza. Neste caso, $50 \%$ para cada um. Isso significa que a incerteza atribuída a características de mercado foi considerada de mesma importância do que as incertezas intrínsecas aos parâmetros que estavam em análise. Logo, para um parâmetro i em uma opção de investimento j, tem-se a Equação (06): 


$$
s_{i}^{2}=\mathrm{x}_{i}\left(0,5 \cdot \mathrm{IG}_{j}+0,5 \cdot \mathrm{IE}_{i}\right)
$$

Onde $\mathrm{s}^{2}, x$, IE são, respectivamente, o desvio padrão, a média e o nível de incerteza específico (em decimal) do parâmetro i e IG é o nível de incerteza geral da opção de investimento j (também em decimal).

Para a utilização do Metódo de Monte Carlo com as considerações anteriores, tornase necessário arbitrar um número de simulações, ou seja, quantos cenários futuros seriam modelados para a continuação da metodologia. A utilização, neste estudo, foi de 10.000 cenários futuros e, para tanto, cada parâmetro de cada uma das alternativas foi simulada 10.000 vezes.

Com todos os valores simulados, para o cálculo do VPL de cada um dos 30.000 fluxos de caixa (10.000 para cada estratégia) que compõem esta análise de investimento, a próxima etapa é a definição de uma TMA. Para tanto, considerou-se o Tesouro Prefixado 2020 (LTN), cujo valor é 7,06\% ao ano, já com os devidos descontos legais.

Os Critérios de Decisão em Condições de Incerteza, logo, são empregados nos dados presentes na Matriz de Decisão de forma a apresentarem, cada um segundo suas diretrizes, a melhor opção de investimento.

\section{RESULTADOS E DISCUSSÕES}

A seguir são apresentados, na Tabela 2, os valores dos níveis de incerteza atribuídos para os parâmetros em questão na análise de investimentos estudada neste trabalho.

TABELA 2 - NÍVEIS DE INCERTEZA GLOBAL E ESPECÍFICO PARA OS PARÂMETROS

\section{$\begin{array}{llll}\text { Parâmetros } & \text { Investimento } 1 & \text { Investimento } 2 & \text { Investimento } 3\end{array}$}

\begin{tabular}{lccc}
\hline Investimento Inicial & $2,5 \%$ & $2,5 \%$ & $2,5 \%$ \\
\hline Demanda por Período & $10 \%$ & $10 \%$ & $10 \%$ \\
\hline Preço de Venda Unitário & $10 \%$ & $10 \%$ & $10 \%$ \\
\hline Custo Matéria Prima Unitário & $5 \%$ & $5 \%$ & $5 \%$ \\
\hline Custo Fornecedor Unitário & $5 \%$ & $5 \%$ & $5 \%$ \\
\hline Nível de Incerteza Global & $7,5 \%$ & $5 \%$ & $10 \%$ \\
\hline
\end{tabular}

FONTE: Os autores (2018)

Com as devidas definições acerca dos níveis de incerteza e determinada a TMA relacionada aos fluxos de caixa, é possível, portanto, calcular os valores de VPL de todas as 
simulações de cada uma das 3 estratégias de investimentos que são analisadas. Assim, é possível estabelecer a Matriz de Decisão, apresentada na Tabela 3, a qual reúne todos os possíveis cenários futuros modelados para cada opção de investimento em análise.

TABELA 3 - MATRIZ DE DECISÃO

\begin{tabular}{cccc}
\hline \multirow{2}{*}{ Cenário } & \multicolumn{3}{c}{ VPL (R\$) } \\
\cline { 2 - 4 } & Investimento 1 & Investimento 2 & Investimento 3 \\
\hline 1 & $2.572 .481,72$ & $3.380 .479,88$ & $2.344 .150,31$ \\
\hline 2 & $2.496 .384,86$ & $2.363 .145,06$ & $3.278 .908,55$ \\
\hline$(\ldots)$ & $(\ldots)$ & $(\ldots)$ & $(\ldots)$ \\
\hline 10000 & $2.663 .464,62$ & $2.192 .786,98$ & $2.590 .487,95$ \\
\hline
\end{tabular}

FONTE: Os autores (2018)

Dos métodos de Critério de Decisão em condições de Incerteza utilizados neste estudo, deve-se destacar que no caso do Critério de Hurwicz e Hurwicz Modificado, analisaram-se os casos em que o investidor tem um perfil mais otimista. Assim sendo, de acordo com Garcia et. al. (2010) e Pérez et. al. (2015), para o melhor dos cenários atribuiuse um peso de $70 \%$ na média para o cálculo do VPL representativo, sendo, portanto, $\alpha=0,7$ aplicado na Equação 03. De forma complementar se avalia, ainda, um outro caso, de pessimismo, onde esse valor foi alterado para $30 \%(\alpha=0,3)$.

Para o Modelo de Amplitude, apresentado pela Equação 05, os valores representativos de VPL foram obtidos utilizando a soma de $80 \%(\beta=0,80)$ para a média e uma penalização equivalente a $20 \%$ da amplitude ao valor do VPL médio das alternativas, como orientado por Ramírez \& García (2003).

Em se tratando do Critério de Savage, elaborou-se a Matriz de Arrependimento, apresentada na Tabela 4 que relacionada as opções de investimento em análise.

TABELA 4 - MATRIZ DE ARREPENDIMENTO

\section{Cenário}

\section{VPL (R\$)}

\section{Investimento $1 \quad$ Investimento 2 Investimento 3}

\begin{tabular}{cccc}
\hline 1 & $1.249 .653,88$ & $619.828,45$ & $1.544 .080,05$ \\
\hline 2 & $1.325 .750,73$ & $1.637 .163,27$ & $609.321,81$
\end{tabular}




\begin{tabular}{cccc}
\hline$(\ldots)$ & $(\ldots)$ & $(\ldots)$ & $(\ldots)$ \\
\hline 10000 & $1.158 .670,97$ & $1.807 .521,35$ & $1.297 .742,41$ \\
\hline
\end{tabular}

FONTE: Os autores (2018)

Então, a partir da Matriz de Decisão (Tabela 3), podem ser observados os resultados da utilização dos Critérios de Decisão em Condições de Incerteza na Tabela 5, a seguir. 
REVISTA LATINO-AMERICANA DE INOVAÇÃO E

ENGENHARIA DE PRODUÇÃO

TABELA 5 - RESULTADOS DA UTILIZAÇÃO DOS CRITÉRIOS DE DECISÃO EM CONDIÇÕES DE INCERTEZA, COMBINADOS COM O MÉTODO DE MONTE CARLO

\begin{tabular}{llllll}
\hline \multirow{2}{*}{ Método } & & \multicolumn{2}{c}{ Cenário } & VPL (R\$) & Opção escolhida \\
\cline { 3 - 5 } & & Investimento 1 & Investimento 2 & Investimento 3 & \\
\hline Maximin & Mais pessimista de cada (Menor) & $1.281 .693,50$ & $1.447 .637,18$ & $1.514 .614,81$ & Investimento 3 \\
\hline Maximax & Mais otimista de cada (Maior) & $3.822 .135,59$ & $4.000 .308,33$ & $3.888 .230,36$ & Investimento 2 \\
\hline \multirow{2}{*}{ Hurwicz } & VPL representativo: maior otimismo & $3.060 .002,96$ & $3.234 .506,98$ & $3.176 .145,69$ & Investimento 2 \\
\cline { 2 - 5 } & VPL representativo: maior pessimismo & $2.043 .826,12$ & $2.213 .438,53$ & $2.226 .699,48$ & Investimento 3 \\
\hline Savage & Maiores arrependimentos de cada & $2.540 .442,10$ & $2.552 .671,14$ & $2.373 .615,55$ & Investimento 3 \\
\hline Laplace & Maiores VPL médios de cada & $2.463 .093,70$ & $2.607 .685,78$ & $2.720 .213,73$ & Investimento 3 \\
\hline $\begin{array}{l}\text { Hurwicz } \\
\text { Modificado }\end{array}$ & VPL representativo: maior otimismo & $2.566 .164,35$ & $2.717 .378,33$ & $2.811 .919,65$ & Investimento 3 \\
\cline { 2 - 5 } $\begin{array}{l}\text { Modelo } \\
\text { Amplitude }\end{array}$ & VPL representativo: maior pessimismo & $2.359 .530,15$ & $2.497 .472,29$ & $2.627 .963,34$ & Investimento 3 \\
\hline & VPL representativo calculado & $3.925 .480,25$ & $4.183 .300,17$ & $4.421 .661,61$ & Investimento 3 \\
\hline
\end{tabular}

FONTE: Os autores (2018)

Rev. Lat.-Am. Inov. Eng. Prod. [Relainep]

Curitiba, Paraná, Brazil v. 7 n. $11 \quad$ p. $47-67 \quad 2019$

DOI: $10.5380 /$ relainep.v7i11.63870 
A seguir, na Figura 1, é apresentada uma ilustração comparativa entre os valores de VPL representativos utilizados para cada um dos Critérios de Decisão em Condição de Incerteza e as suas respectivas opções de investimento.

\section{FIGURA 1 - REPRESENTAÇÃO GRÁFICA DE VPL REPRESENTATIVO}

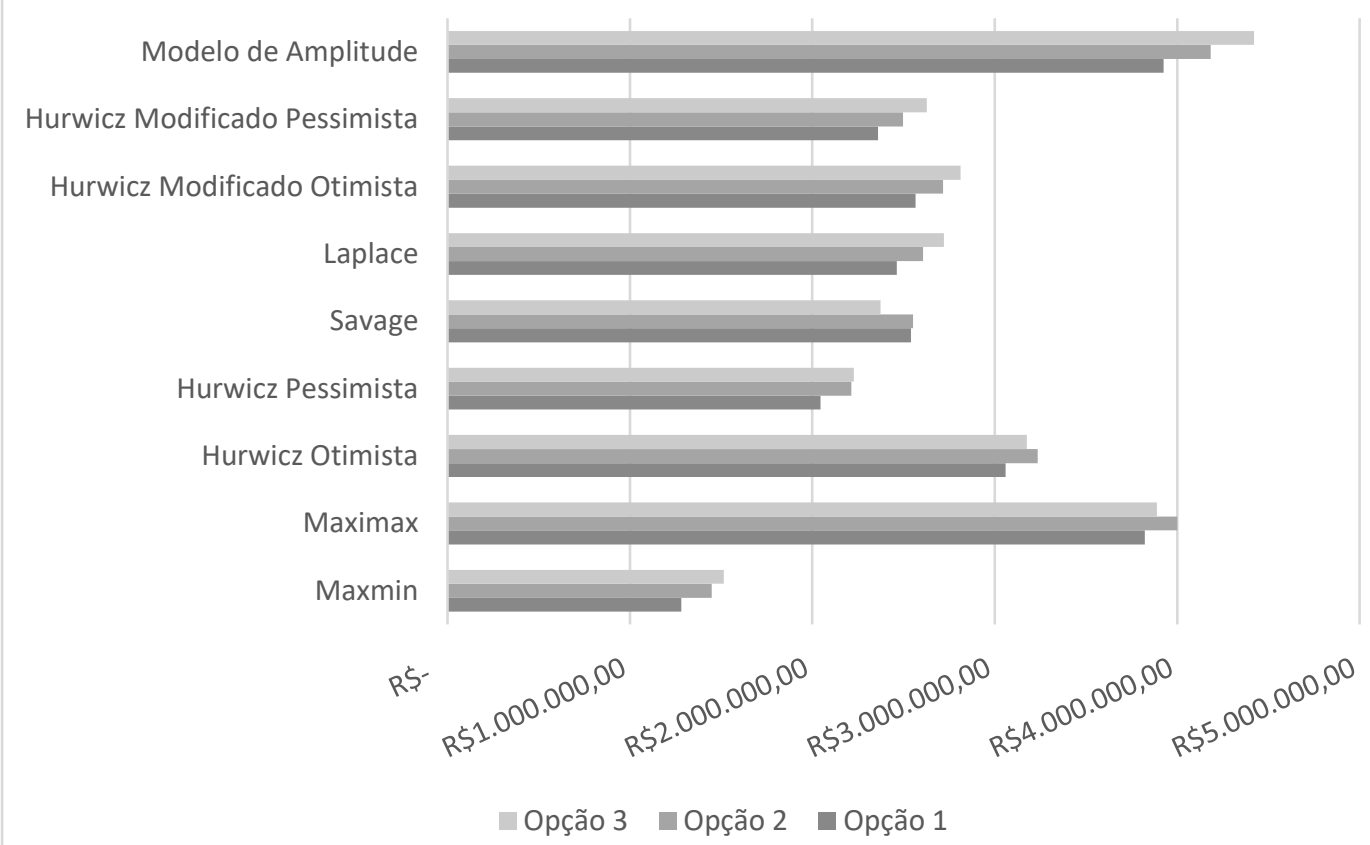

FONTE: Os autores (2018)

Com exceção dos critérios Maximax, Savage e Hurwicz Otimista, todos os demais indicaram que a melhor opção de investimento, considerando os dados gerados pelo Método de Monte Carlo, é a opção 3.

Constatou-se que, alguns dos Critérios de Decisão em Condições de Incerteza podem se apresentar menos adequados por considerarem como referência apenas um ou dois valores dos milhares calculados pelo Método de Monte Carlo. Tal situação ocorre com os critérios: Maximin, Maximax, Hurwicz e Savage.

Já os métodos de Laplace, Hurwicz Modificado e, em especial, o Modelo de Amplitude, no entanto, não apresentam tais dificuldades exatamente por trabalharem com valores médios, considerando assim, todos os valores gerados pelo Método de Monte Carlo.

O Modelo de Amplitude, ainda, acaba por se destacar dos demais por, além de conseguir incorporar os diversos valores simulados na modelagem, ainda penalizar as alternativas que possuem um maior grau de incerteza, através da consideração da amplitude 
dos valores apresentados.

\section{CONCLUSÃO}

A pesquisa aqui relatada apresentou uma possibilidade de utilizar um método direcionado para a análise de investimentos, viável e aplicável em diversas áreas e em qualquer situação, onde se tem um fluxo de caixa que dependente de variáveis incertas ou até mesmo investimentos com horizonte de planejamento diferentes.

As incertezas inseridas na modelagem do problema, considerando o futuro indeterminado, podem ser consideradas por meio dos valores bases (média e desvio padrão) aplicados nos parâmetros incertos a serem simulados pelo Método de Monte Carlo ou pela escolha de outros critérios para se utilizar como referência.

Em relação aos valores bases, média e desvio padrão, recomenda-se que sejam atribuídos como resultado de um estudo do mercado em que o produto, material de interesse do investimento, está inserido e, também, dos parâmetros que, por si só apresentam diferentes níveis de certeza.

De forma parecida, parâmetros estabelecidos para sofrer variação apresentam diferentes níveis de incerteza relacionados a si próprios. Por exemplo, os custos relacionados a variação de preços dos fornecedores provavelmente apresentem um maior nível de segurança quanto a estimativa de valores, independentemente do nível de estudo relacionado ao mercado, se comparado a incerteza relativa a demanda que o produto apresentará. Por essa razão, além da consideração da incerteza nos desvios padrões, relacionada ao conhecimento do mercado, a segurança relativa aos parâmetros em análise também pode ser representada nesses.

A forma de atribuir pesos nas quantificações dos dois tipos de incertezas aqui apresentados pode ainda variar. Nesta aplicação, foram estipuladas importâncias iguais para ambas, mas dependendo do mercado em análise e dos conhecimentos relativos a eles que o investidor apresentar, tal proporção pode ser alterada.

Apesar da grande simplicidade relativa a maioria dos Critérios de Decisão em Condições de Incerteza, estes apresentaram-se capazes de direcionar o investidor a uma tomada de decisão com embasamento, considerando um futuro probabilisticamente indeterminado. Com isto posto, recomenda-se que, ao utilizar o Método de Análise de 
Investimentos proposto por esta pesquisa, considere-se um maior peso a indicação dada pelo Modelo de Amplitude, seguida pelas informações fornecidas pelos métodos de Laplace e de Hurwicz Modificado, e, com menor significância aos demais apresentados.

\section{REFERÊNCIAS}

BRANDIMARTE, P. Handbook in Monte Carlo Simulation Applications in Financial Engineering, Risk Management, and Economics. Italy: Wiley, 2014.

BUCHNER, A. Risk Management for Private Equity Funds. Journal of Risk, 19(6), 132, 2017. https://doi.org/10.21314/JOR.2017.363

CAMARGO, C. Análise de Investimentos e Demonstrativos Financeiros ( 1 ed.). Curitiba: Intersaberes, 2007.

CASAROTTO FILHO, N., \& KOPITTKE, B. H. Análise de Investimentos. São Paulo: Atlas, 2006.

CHOBOT, K. Multi-Criteria Decision-Making in Management under Conditions of Risk and Uncertainty. In Anais 22nd International Conference On Metallurgy And Materials. Czech Republic, 2013.

FONSECA, Y. D. Técnicas de avaliação de investimentos: uma breve revisão da literatura. Cadernos de Análise Regional. (2003). Disponível em <http://www.infinitaweb.com.br/albruni/artigos/a0303_CAR_AvalInvest.pdf > Acesso em: Novembro de 2017

GARCIA, S., LUSTOSA, P. R. B.,\& BARROS, N. R. Aplicabilidade do Método de Simulação de Monte Carlo na Previsão dos Custos de Produção de Companhias Industriais: o Caso da Companhia Vale do Rio Doce. Revista de Contabilidade e Organizações, 4(10), 152-173, 2010.

GASPARS-WIELOCH, H. Modifications of the Hurwicz's Decision Rule. Central European Journal of Operations Research, 22(4), 779-794, 2014. https://doi.org/10.1007/s10100-013-0302-y

GALESNE, A., FENSTERSEIFER, J. E., LAMB, R. Decisões de Investimentos da Empresa. São Paulo: Atlas, 1999.

GROENEWALD, M. E.,\& PRETORIUS, P. D. Comparison of Decision-Making Under Uncertainty Investment Strategies With the Money Market. Journal of Financial Studies and Research, 2011(2011), 1-16, 2011. http://dx.doi.org/10.5171/2011.373376

HAN, Z., PORRAS-ALVARADO, J. D., SUN, J.,\& ZHANG, Z. Monte Carlo Simulation-Based Assessment of Risks Associated with Public-Private 
Partnership Investments in Toll Highway Infrastructure. Transportation

Research Record, 2670, 59-67, 2017. https://doi.org/10.3141/2670-08

JUNIOR, C. K.; ZARPELLON, F. R.; DALAZEN, L. L.; SOUZA, A. A cultura da Ervamate (Ilex paraguariensis) em sistema de cultivo convencional e orgânico como alternativa de renda ao pequeno proprietário rural. In Anais: XXIV Congresso Brasileiro de Custos. 2017. Disponível em: https://www.researchgate.net/publication/320765832_A_cultura_da_Ervamate_Ilex_paraguariensis_em_sistema_de_cultivo_convencional_e_organico_com o_alternativa_de_renda_ao_pequeno_proprietario_rural. Acesso em: Novembro de 2017.

LAURENCEL, L. C. \& REZENDE FILHO, M. Engenharia Financeira: fundamentos para avaliação e seleção de projetos de investimentos e tomada de decisão. 1 ed. Rio de Janeiro: LTC, 2013.

LIMA, EURICO CAVALCANTI PINCOVSKY DE; VIANA, J. C.; LEVINO, N. A.; MOTA, C. M. M. Simulação de monte carlo auxiliando a análise de viabilidade econômica de Projetos. In Anais IV Congresso Nacional de Excelência em Gestão. p. 1-13. Niterói: CNEG, 2008.

MARTINS, M. S. Modelagem para Negócios Sustentáveis. In Anais do Encontro de Ensino, Pesquisa e Extensão, 9 (n Especial). Presidente Prudente: Colloquium Humanarum, 2012.

MELliCHAMP, D. A. New Discounted Cash Fow Method: Estimating Plant Profitability at the Conceptual Design Level While Compensating for Business Risk/Uncertainty. Computers and Chemical Engineering, v. 48, p. 251-263, 2013. http://dx.doi.org/10.1016/j.compchemeng.2012.08.012

O’DONNELL, B. R., HICKNER, M. A., \& BARNA, B. A. Economic Risk Analysis Using Analytical and Monte Carlo Techniques. Chemical Engineering Education, 36(2), 94-99, 2002.

PAŽEK, K.,\& ROZMAN, C. Decision Making Under Conditions of Uncertainty in Agriculture: a Case Study of Oil Crops. Poljoprivreda, 15(1), 45-50, 2009.

PÉREZ, D. E., HERNÁNDEZ, J. G., GARCÍA, M. J., \& HERNÁNDEZ, G. J. HURWICZ. Method modified and The Amplitude Model (TAM). In Anais do Global Business and Technology Association. 1, p. 559 - 566. Lisboa: GBATA, 2015 .

RAMÍREZ, J. G. H., \& GARCÍA., M. J. Toma de Decisiones Bajo Incertidumbre Considerando la Dispersion. In Anais da XXVII Conferencia Latinoamericana de Informática, 27. Mérida, 2001.

RAMÍREZ, J. G. H., \& GARCÍA, M. J. Analysis of the beta ( $\beta$ ) factor, in The Amplitude Model (TAM). Revista de Matemática: Teoria y Aplicaciones. 1-2, (10), 187 199, 2003. 
RYBA, A., LENZI, E. K., LENZI, M. K. Elementos de Engenharia Econômica (2 ed.). Curitiba: Intersaberes, 2016.

SHARMA, K. R. An Introduction to Engineering Economics. New York: Momentum Press, 2015.

SOBOL, I. O Método de Monte Carlo.Moscou: Mir Moscou, 1972.

SOUZA, F. A.; SANTOS, J. C.; SENA, A. L. S.; ELLERES, A. S. Avaliação Econômica e de Risco em Sistema de Produção de Soja na Região de Santarém, Estado do Pará. In: $2^{\circ}$ Seminário de Iniciação Científica e $4^{\circ}$ Seminário de Pós-Graduação da Embrapa Amazônia Oriental. 2016. Disponível em: https://www.embrapa.br/web/mobile/publicacoes/-/publicacao/1053911/avaliacaoeconomica-e-de-risco-em-sistema-de-producao-de-soja-na-regiao-de-santaremestado-do-para. Acesso em Novembro de 2017. 